\title{
Evidence for a protein related immunologically to the jaagsiekte sheep retrovirus in some human lung tumours
}

\author{
M. De las Heras*, S.H. Barsky**, P. Hasleton***, M. Wagner ${ }^{+}$, E. Larson ${ }^{++}$, J. Egan***, A. Ortin*, \\ J.A. Gimenez-Mas" ${ }^{\#}$ M. Palmarini ${ }^{\S}$, J.M. Sharp ${ }^{\S}$
}

Evidence for a protein related immunologically to the jaagsiekte sheep retrovirus in some human lung tumours. M. De las Heras, S.H. Barsky, P. Hasleton, M. Wagner, E. Larson, J. Egan, A. Ortin, J.A. Gimenez-Mas, M. Palmarini, J.M. Sharp. (C) ERS Journals Ltd 2000. ABSTRACT: Human bronchioloalveolar carcinoma (BAC) is a lung cancer, morphologically similar to an endemic contagious lung neoplasm of sheep called sheep pulmonary adenomatosis (SPA) or jaagsiekte. SPA is caused by an exogenous type $B / D$ retrovirus (jaagsiekte sheep retrovirus (JSRV)), which prompted the present study to obtain evidence of a retrovirus in BAC.

A panel of 249 human lung tumours, 21 nontumour lung lesions, four normal lung tissues, 23 adenocarcinomas from other organs and a cell line expressing a human endogenous retrovirus protein was examined immunohistochemically using a rabbit antiserum directed against the JSRV capsid protein.

Specific staining was detected only in the cytoplasm of recognizably neoplastic cells in the pulmonary alveoli of 39 of $129(30 \%)$ BACs, 17 of $65(26 \%)$ lung adenocarcinomas and two of seven large cell carcinomas. The remaining samples were negative.

These results support the hypothesis that some human pulmonary tumours may be associated with a jaagsiekte sheep retrovirus-related retrovirus, warranting further studies.

Eur Respir J 2000; 15: 330-332. *Departamento de Patologia Animal, Univer-
sidad de Zaragoza, Saragossa, Spain, ${ }^{\S}$ More-
dun Research Institute, Edinburgh, UK,
***North West Lung Centre, Wythenshawe
Hospital, Manchester, UK, '+ Dept of Path-
ology, University of Uppsala, Uppsala,
Sweden, "Hospital General Miguel Servet,
Saragossa, Spain, ${ }^{+}$Dept of Pathology, Uni-
versity of Köln, Cologne, Germany, **Uni-
versity of California, Los Angeles School of
Medicine, Los Angeles, CA, USA.

Correspondence: J.M. Sharp, Moredun Research Institute, Pentlands Science Park, Bush Loan, Edinburgh, Midlothian EH26 OPZ UK. Fax: 441314456111

Keywords: Bronchioloalveolar carcinoma, jaagsiekte, lung cancer, retrovirus, sheep pulmonary adenomatosis

Received: April 211999

Accepted after revision March 102000

Funding for these studies was provided by the Scottish Executive, Rural Affairs Dept (ROAME MRI/027/95), the Commission for the European Communities (AIR3-CT94084), the Spanish Government (CICYT AGF 960535-C02-01), Gobierno de Aragón(PO5/97), the Tobacco-Related Disease Research Program (8IT-0046) and the Nickoll Family Foundation.
Human bronchioloalveolar carcinoma (BAC) is a subset of pulmonary adenocarcinoma (ADC) arising from type II pneumocytes or Clara cells [1]. The disease shows a variety of unique features and is distinguished from other pulmonary tumours, including $\mathrm{ADC}$, by its multifocal location in the peripheral parenchyma, the absence of a central bronchogenic source or extrathoracic adenocarcinoma and its apparent intra-alveolar spread with no disruption of the intra-alveolar space $[2,3]$. Histological features have allowed further classification of BAC into mucinous, nonmucinous and mixed forms [1]. The prevalence of BAC is low, but appears to be increasing dramatically in the USA and Japan $[4,5]$. In contrast to most other malignant lung tumours, the epidemiology of BAC indicates only a weak association with smoking [3, 4].

The histology and the ultrastructure of BAC closely resembles that of an endemic contagious disease of sheep called sheep pulmonary adenomatosis (SPA) or jaagsiekte [6]. An exogenous type B/D retrovirus (jaagsiekte sheep retrovirus (JSRV)) is the causal agent of SPA [7] and appears to replicate mainly in the transformed epithelial cells
[8]. Renewed speculation regarding the involvement of a retrovirus in BAC [9] has been supported by the association of retroviruses, other than human immunodeficiency virus and human T-cell leukaemia virus, with several diseases of humans, such as seminoma [10], undifferentiated germ cell tumours [11], multiple sclerosis [12], acute-onset type I diabetes [13] and Sjögren's syndrome [14]. In order to address this issue, a panel of human lung tumours and relevant nontumour lung lesions were examined immunohistochemically using an antiserum to JSRV capsid protein (JSRV-CA).

\section{Materials and methods}

Paraffin-embedded samples (274) from patients with BAC, other pulmonary malignancies, nonmalignant pulmonary lesions and normal lung were examined (table 1). Samples were obtained from patients in Germany, Japan, Mexico, Spain, Sweden, the UK and the USA. All tumours were classified in accordance with the 1999 World Health Organization classification [2]. The final panel 
Table 1. - Demonstration of an antigen related to jaagsiekte sheep retrovirus in human tissues

\begin{tabular}{|c|c|c|c|c|c|c|}
\hline \multirow{2}{*}{ Type of tumour } & \multicolumn{6}{|c|}{ Proportion of antigen-positive tissues } \\
\hline & Germany* & Spain & Sweden & UK & USA & Total \\
\hline Bronchioloalveolar carcinoma & $6 / 22$ & $2 / 9$ & $3 / 7$ & $3 / 9$ & $25 / 82$ & $39 / 129$ \\
\hline Adenocarcinoma & $0 / 5$ & $1 / 4$ & $1 / 2$ & $3 / 18$ & $12 / 46$ & $17 / 65$ \\
\hline Large cell carcinoma & & & $1 / 1$ & & $1 / 6$ & $2 / 7$ \\
\hline Squamous cell carcinoma & & $0 / 8$ & $0 / 3$ & $0 / 9$ & $0 / 21$ & $0 / 41$ \\
\hline Carcinoid & & & $0 / 1$ & & $0 / 1$ & $0 / 2$ \\
\hline Large cell neuroendocrine carcinoma & & & & & $0 / 3$ & $0 / 3$ \\
\hline Small cell carcinoma & & & $0 / 2$ & & & $0 / 2$ \\
\hline Nonpulmonary tumours & & & & & $1 / 23$ & $1 / 23$ \\
\hline Primary pulmonary hypertension & & & & $0 / 9$ & & $0 / 9$ \\
\hline Cryptogenic fibrosing alveolitis & & & & $0 / 11$ & & $0 / 11$ \\
\hline Methotrexate-induced fibrosis and metaplasia & & & & & $0 / 1$ & $0 / 1$ \\
\hline Normal lung & & & & & $0 / 4$ & $0 / 4$ \\
\hline
\end{tabular}

*: includes samples from Mexico and Japan.

comprised: 129 BACs, 65 ADCs, 41 squamous cell carcinomas (SCCs), two small cell lung cancers (SLCs), two typical carcinoids (CAs), seven large cell carcinoma (LCCs), and three large cell neuroendocrine carcinomas. BACs from the USA were subclassified into 13 mucinous, 60 nonmucinous, and nine mixed mucinous/nonmucinous forms. All lung tumours examined were primary pulmonary malignancies. All patients showed no evidence of ADC or other tumours in a nonpulmonary organ. Nontumour pulmonary lesions were obtained from nine patients with primary pulmonary hypertension (PPH), 11 cryptogenic fibrosing alveolitis, one methotrexate-induced fibrosis and bronchioloalveolar metaplasia (ME) and four normal lung (NL) (table 1). Twenty three ADCs of nonpulmonary origin were also examined, comprising nine ductal carcinomas of the breast, nine prostatic ADCs, three pancreatic ADCs and two pulmonary metastases from nonpulmonary primary tumours.

Tissue sections (4-6 $\mu \mathrm{m})$ from each sample were examined for JSRV-related antigens using a well-characterized specific rabbit antiserum directed against JSRV capsid protein (JSRV-CA) by means of the immunohistochemical protocol reported previously [8], except that an antigenretrieval step was included. This additional procedure consisted of heating the sections twice in an 800-W microwave for $7 \mathrm{~min}$, which had been demonstrated to be appropriate for JSRV-CA in SPA tumours. SPA tumour was used as a positive control and unaffected lung as a negative control. To exclude the possibility that the antiserum directed against JSRV-CA detected related human endogenous retroviruses (HERV) e.g. HERV-K, the GH cell line, FA2/3.7.97 [15], which constitutively expresses HERV-K gag, was included.

\section{Results}

Immunohistochemical analysis with JSRV-CA antiserum revealed a positive reaction in 58 of $249(23 \%)$ primary pulmonary tumours, zero of 25 nontumour pulmonary tissues and only one of 23 (4.3\%) ADCs of nonpulmonary origin (table 1). The positive reaction was confined to the epithelial tumour cells and showed the same characteristics as that obtained with SPA tumours (fig. 1). No positive material was detected in interstitial cells or observed extracellularly. Preinoculation rabbit antiserum did not stain any sample.
Of the 58 of 249 positive pulmonary tumours, 39 of 129 $(30.2 \%)$ were BACs, 17 of $65(26.2 \%)$ were ADCs and two of seven $(28.6 \%)$ LCCs (table 1). Of the 82 BAC samples from the USA, positive reactions were observed in 18 of $60(30 \%)$ nonmucinous, two of $13(15 \%)$ mucinous and five of nine $(56 \%)$ mixed forms.

No positive staining was observed in any of the other 51 primary lung tumours nor in the 25 nontumour lesions and normal lung tissues (table 1).

In the group of 23 primary nonpulmonary ADCs, one ductal carcinoma of the breast gave weak specific positive

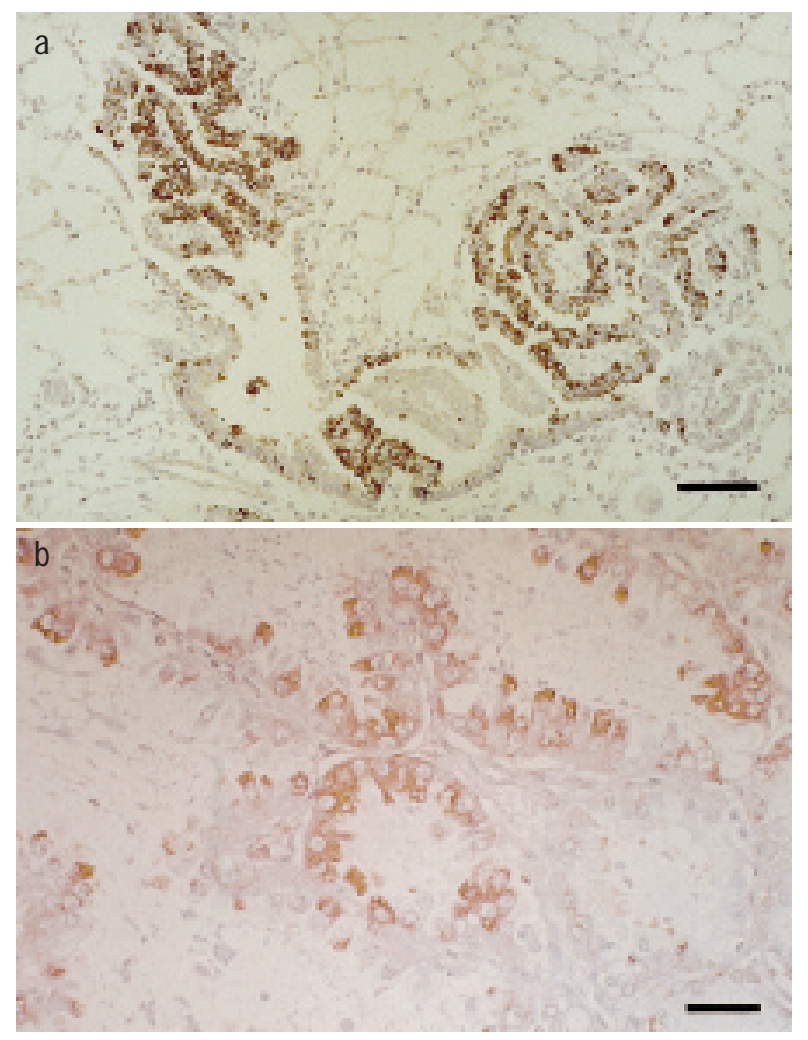

Fig. 1. - Immunohistochemical analysis performed wih antiserum directed against jaagsiekte sheep retrovirus capsid protein. The specific dark brown staining is confined to the cytoplasm of epithelial tumour cells: a) sheep pulmonary adenomatosis; and b) bronchioloalveolar carcinoma. (Internal scale bars $=45 \mu \mathrm{m}$ (a); $60 \mu \mathrm{m}$ (b).) 
staining; the remaining 22 specimens were negative, as was the cell line GH (FA 2/33.7.97) that constitutively expresses HERV-K gag.

Statistical analysis of the results showed that there were no differences between the numbers of positive BACs, ADCs and LCCs $(p=0.15-0.84)$ but each of these was significantly greater than the number of SCCs $(\mathrm{p}=0.0001-$ $0.006)$, nontumour pulmonary tissues $(p=0.0005-0.02)$ and primary nonpulmonary ADCs $(\mathrm{p}=0.0009-0.02)$.

\section{Discussion}

Attention has been drawn recently to pulmonary ADCs, particularly because of their increasing prevalence, despite only a weak association with cigarette smoking $[4,5,16]$. The multifocal presentation of BAC, especially the nonmucinous and mixed types, and data pointing to a multiclonal origin in some cases, has rekindled the view that some of these tumours may be due to a carcinogenic agent or oncogenic virus [17].

The present study has provided evidence for an antigen related to an ovine retrovirus, JSRV, in three forms of primary pulmonary carcinoma, BAC, ADC and LCC. The pattern of immunohistochemical staining was identical to that observed in the ovine tumour, SPA, using the same well-characterized serum [8], and supports the view that the antigen in the human tumours may be a retrovirus protein. The antigen was detected in $26-30 \%$ of these three tumours, which was significantly more than it was detected in 74 specimens from other pulmonary and nonpulmonary tumours or 25 samples of nontumour lesions $(\mathrm{p}<0.02)$, most notably normal and metaplastic lungs, such as PPH and ME.

The above positive correlation suggests that a retrovirus may be associated with the neoplastic process in some tumours or represent reactivation/upregulation of an endogenous retrovirus. Human retroviruses, related phylogenically to JSRV, have been linked with various diseases (see "Introduction" section); therefore, the antigen detected in BAC, ADC and LCC may reflect expression of one of these retroviruses or a previously undescribed virus. The HERV most closely related to JSRV is HERV-K, which is expressed in the cytoplasm of seminoma tumours and teratocarcinoma cell lines $[10,15]$. However, the observed staining is unlikely to result from expression of HERV-K proteins since no specific reaction was obtained with a cell line expressing HERV-K gag proteins.

Research to extend these preliminary findings, and confirm any association of a retrovirus with human lung tumours, should provide additional complementary molecular and virological data.
Suzumiya from the Dept of Pathology, Fukuoka University Medical School, Japan and C.R.F. Krueger from the Dept of Pathology, Universität Köln, Cologne, Germany for provision of some specimens.

\section{References}

1. Travis WD, Colby TV, Borrin B, Shimosato Y, Brambilla E. Histological typing of lung and pleural tumours. International histological classification of tumours. 1999; 3rd ed. Geneva. World Health Organization.

2. Schraufnagel D, Peloquin A, Paré JAP. Differentiating bronchioloalveolar carcinoma from adenocarcinoma. Am Rev Respir Dis 1982; 125: 74-79.

3. Hill CA. Bronchioloalveolar carcinoma: A review. Radiology 1984; 150: 74-79.

4. Barsky SH, Cameron R, Ossan KE, Tomita D, Holmes EC. Rising incidence of bronchioloalveolar lung carcinoma and its unique clinicopathologic features. Cancer 1994; 73: 1163-1170.

5. Auerbach O, Garfinkel L. The changing pattern of lung adenocarcinoma. Cancer 1991; 68: 1973-1977.

6. Bonne C. Morphological resemblance of pulmonary adenomatosis (Jaasiekte) in sheep and certain cases of cancer of the lung in man. Am J Cancer 1939; 35: 491501.

7. Palmarini M, Sharp JM, De las Heras M, Fan HY. Jaagsiekte sheep retrovirus is necessary and sufficient to induce a contagious lung cancer in sheep. J Virol 1999; 73: 6964-6972.

8. Palmarini M, Dewar P, De las Heras M, Inglis NF, Dalziel RG, Sharp JM. Epithelial tumour cells in the lungs of sheep with pulmonary adenomatosis are major sites of replication of Jaagsiekte retrovirus. J Gen Virol 1995; 76: 2731-2737.

9. Cremer K, Gruber J. Animal models of retrovirusassociated malignancies. Vet Pathol 1992; 29: 572-578.

10. Sauter M, Schommer S, Kremmer E, Remberger K, Dölken G, Müller-Lantzsch N. Human endogenous retrovirus K-10: expression of gag protein and detection of antibodies in patients with seminomas. J Virol 1995; 69: 414-421.

11. Herbst H, Santer M, Müller-Lantzsch N. Expression of human endogenous $\mathrm{K}$ elements in germ cell and tropholastic tumours. Am J Pathol 1996; 149: 1727-1735.

12. Perron H, Garson JA, Bedin F, et al. Molecular identification of a novel retrovirus repeatedly isolated from patients with multiple sclerosis. Proc Natl Acad Aci USA 1997; 94: 7583-7588.

13. Conrad B, Weissmahr RN, Boni J, Arcari R, Schupbach J, Mach B. A human endogenous retroviral superantigen as candidate autoimmune gene in type I diabetes. Cell 1997; 90: 303-313.

14. Griffiths DJ, Venables PJ, Weiss RA, Boyd MT. A novel exogenous retrovirus sequence identified in humans. $J$ Virol 1997; 71: 2866-2872.

15. Löwer J, Löwer R, Frank H, Harzmann R, Kurth R. Human teratocarcinomas cultured in vitro produce unique retrovirus-like viruses. J Gen Virol 1984; 65: 887-898.

16. Morton WE, Treyve EL. Histologic differences in occupational risks of lung cancer incidence. Am J Ind Med 1982; 3: 441-457.

17. Barsky SH, Grossman DA, Ho J, Holmes EC. The multifocality of bronchioloalveolar lung carcinoma: evidence and implications of a multiclonal origin. Mod Pathol 1994; 7: 633-640. 